Pharmaceutical
companies have
not realized the
promised
productivity
gains from novel
research
technologies.
Discovery
'industrialization'
also demands
profound
changes to both
people skills and
organization for
the benefits to
be delivered.

Richard Archer CEO, The Automation Partnership Ltd, York Way, Royston, Hertfordshire SG8 5WY, UK. e-mail: richard.archer@ automationpartnership.com

\title{
CAN MASTER CRAFTSMEN BE MASS-PRODUCED?
}

This question was undoubtedly being asked by the eighteenth century Trade Guilds in response to The Industrial Revolution. This represented a complete transformation of manufacturing, in which processes moved from discrete cottage-scale operations to a profoundly larger, distributed approach. Such a radical overhaul inevitably produced detractors. Arguably, those with the most to lose were the Master Craftsmen, who represented the backbone of pre-Industrial Revolution manufacturing. No doubt many of them resented the move to a model in which groups of relatively unskilled workers could produce a product in less time, and to better accuracy and lower unit cost than they could ever achieve.

How is this relevant to employment in the world of modern drug discovery? Perhaps more than is immediately apparent. The perception of drug discovery today is of an industry that requires both superior scientific skills and high technology. These new technologies include combinatorial chemistry, high-content cellular and homogenous assays, and high-throughput screening (HTS) process automation (FIG. 1). The past decade has seen a three-orderof-magnitude increase in the peak attainable throughputs of HTS facilities that are, at least, reported at conferences. However, there is a belief, shared in higher management circles, that perhaps all is not as it should be on the HTS floor. Indeed, the number of new chemical entities (NCEs) being delivered by the top pharma companies is insufficient to sustain shareholder expectations, and the upstream pipeline shows only marginal improvement.

There is a push from the present incumbents for yet another round of significant technology investments in order to fuel the provision of 3-5 NCEs per pharma company, per year. Indeed 'new technology evaluation' is seen as a goal in itself for many. The dichotomy is a world in which the Master Craftsman (or screener) is responsible for running a metric-based screening facility 24 hours per day, while still providing the intellectual curiosity behind unravelling new scientific phenomena. This is probably not sustainable. Closer examination shows that many of the current generation of screening technology platforms have much lower utilization rates than might be expected (perhaps only 5\%). The reasons for this have more to do with failures in process management than with the performance of the technology platforms themselves. The received wisdom that 'science cannot be managed' has the net impact that the achieved output of screening facilities bears little relation to their theoretical output capacity.

Some forward-looking organizations are now beginning to comprehend that good science and new technologies alone are no longer sufficient to deliver the desired goals. They are looking hard at how to control processes that were previously considered ungovernable. This requires a different mix of skills and aptitudes from those that typically formed the recruitment basis for a pharmaceutical researcher. In future, academic backgrounds in biophysics, molecular biology or genetics will no longer be the sole requirement. Instead, the industry will be looking elsewhere to include people with experience and core skills in process management, production control, supply-chain management and engineering. A corollary of this change is the desire to move many postgraduate staff out of process-driven positions and back to the world of target identification, for which a high skill level is a prerequisite requirement. The parallels with the Master Craftsmen are too strong to be ignored. The years of training necessary to secure a Ph.D. self-select those interested in the observation of exceptional cases, as opposed to those dedicated to the maintenance of production norms.

Inevitably, for this transition to be successful, some ingrained preconceptions must be altered. However, some of the main pharma companies have recognized that the lack of this skill mix is rate limiting, and are now recruiting to this new model. Those companies with a strong engineering heritage are now transferring technical project expertise across from their manufacturing operations. Others are building their own multi-skilled teams by recruitment and using specialist advisors to specify and interview candidates for these new roles.

For the life scientist, this means keeping an open and curious mind about methods that are adopted in apparently unrelated areas of industry. Life scientists who can bring some additional engineering, project control or operational management skills will be able to command a substantial premium in this emerging area. Equally, those who can operate effectively in a multidisciplinary team will be the user contact of choice for the engineers and managers who are needed to deliver these projects. These skills can be acquired by the committed, through either specialist technical courses or broader qualifications, such as an MBA. This is not a mandate for having fun with robots, however. The process has to be at a higher level than mere technical tinkering to give the scale of result required.

Richard Archer

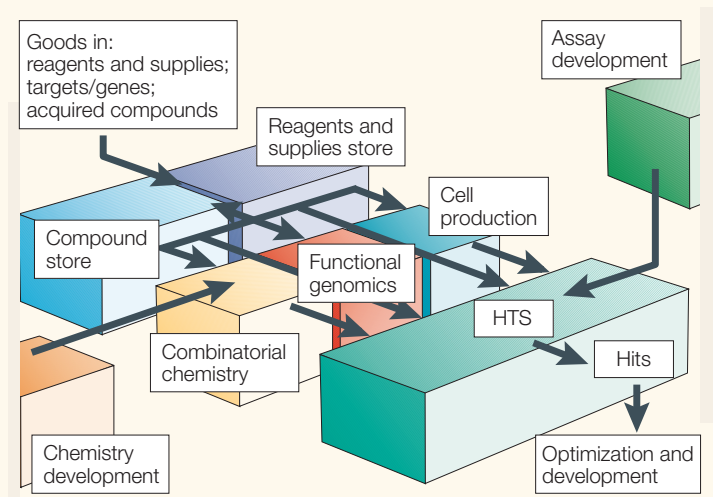

Figure 1 | The drug discovery factory. 